\title{
Graph searching, elimination trees, and a generalization of bandwidth
}

\author{
Fedor V. Fomin Pinar Heggernes Jan Arne Telle \\ Department of Informatics, University of Bergen, N-5020 Bergen, Norway \\ \{fomin, pinar, telle\}@ii.uib.no
}

\begin{abstract}
The bandwidth minimization problem has a long history and a number of practical applications. In this paper we introduce a natural extension of bandwidth to partially ordered layouts. We consider this extension from three main viewpoints: graph searching, tree decompositions and elimination orderings. The three graph parameters pathwidth, profile and bandwidth related to linear layouts can be defined by variants of graph searching using a standard fugitive. Switching to an inert fugitive, the two former parameters are extended to treewidth and fill-in, and our first viewpoint considers the analogous tree-like extension that arises from the bandwidth variant. Bandwidth also has a definition in terms of ordered path decompositions, and our second viewpoint extends this in a natural way to ordered tree decompositions. In showing that both extensions are equivalent we employ the third viewpoint of elimination trees, as used in the field of sparse matrix computations. We call the resulting parameter the treespan of a graph and prove some of its combinatorial and algorithmic properties.
\end{abstract}

\section{Motivation through graph searching games}

Different versions of graph searching has been attracting the attention of researchers from Discrete Mathematics and Computer Science for a variety of elegant and unexpected applications in different and seemingly unrelated fields. There is a strong resemblance of graph searching to certain pebble games [17] that model sequential computation. Other applications of graph searching can be found in VLSI theory since this game-theoretic approach to some important parameters of graph layouts such as the cutwidth [23], the topological bandwidth [22], the bandwidth [9], the profile [10], and the vertex separation number [8] is very useful for the design of efficient algorithms. There is also a connection between graph searching, pathwidth, and treewidth, parameters that play an important role in the theory of graph minors developed by Robertson \& Seymour $[3,7,27]$. Furthermore, some search problems have 
applications in problems of privacy in distributed environments with mobile eavesdroppers ('bugs') [13].

In the standard node-search version of searching, a single searcher is placed at a vertex of a graph $G$ at every move, while from other vertices searchers are removed (see e.g. [17]). The purpose of searching is to capture an invisible fugitive moving fast along paths in $G$. The fugitive is not allowed to run through the vertices currently occupied by searchers. So the fugitive is caught when a searcher is placed on the vertex it occupies, and it has no possibility to leave the vertex because all the neighbors are occupied (guarded) by searchers. The goal of search games is to find a search strategy to guarantee the fugitive's capture while minimizing some resource usage.

Because the fugitive is invisible, the only information the searchers possess are the previous search moves that may give knowledge about subgraphs where the fugitive cannot be present. This brings us to the interesting interpretation of the search problem [3] as the problem of fighting against damage spread in complex systems, e.g. the spread of a mobile computer virus in networks. Initially all vertices are viewed as contaminated (infected by a virus or damaged) and a contaminated vertex is cleared once it is occupied by a searcher (checked by an anti-virus program). A clear vertex $v$ is recontaminated if there is a path without searchers leading from $v$ to a contaminated vertex. In some applications it is required that recontamination should never occur and in this case we are interested in the so-called 'monotone' searching. For most of the search game variants considered in the literature it can be shown, sometimes by very clever techniques, that the resource usage does not increase in spite of this constraint $[4,7,12,17,18]$. The 'classical' goal of the search problem is to find the search program such that the maximum number of searchers in use at any move is minimized. The minimum number of searchers needed to clear the graph is related to the parameter called pathwidth. Dendris et al. [7] studied a variation of the node-search problem with inert, or lazy, fugitive. In this version of the game the fugitive is allowed to move only just before a searcher is placed on the vertex it occupies. The smallest number of searchers needed to find the fugitive in this version of searching is related to the parameter called treewidth [7].

Another criteria of optimality in node-searching, namely search cost was studied in [10]. The cost of a search program is defined as the sum of the number of searchers in use over all moves of this program. Then the goal is to find a search program of the minimum cost. The search cost of a graph is equal to the interval completion number, or profile, which is the smallest number of edges in any interval supergraph of the given graph. Looking at the monotone search cost version but now with an inert fugitive, it is easy to see that this parameter is equal to the smallest number of edges in the chordal supergraph of a given graph, so called fill-in, and we give a formal proof of this fact in Theorem 7.1. We thus have the following elegant relation: the parameters related to standard node searching (pathwidth, profile) expressible in terms of 
interval completion problems, correspond in inert fugitive searching to chordal completion problems (treewidth, fill-in).

In this paper we want to minimize the maximum length of time (number of intermediate moves) during which a searcher occupies a vertex. A similar problem for pebbling games (that can be transferred into search terms) was studied by Rosenberg \& Sudborough [28]. In terms of monotone pebbling (i.e., no recontamination allowed) this becomes the maximum lifetime of any pebble in the game. It turned out that this parameter is related to the bandwidth of a graph $G$, which is the minimum over all linear layouts of vertices in $G$ of the maximum distance between indices of adjacent vertices. The following table summarizes the knowledge about known relations between graph monotone searching and graph parameters.

\begin{tabular}{|c|c|c|c|}
\hline & Number of Searchers & Cost of Searching & Occupation Time \\
\hline Standard Search & pathwidth [17] & profile [10] & bandwidth [28] \\
Inert Search & treewidth [7] & fill-in [11] & $?$ \\
\hline
\end{tabular}

One of the main questions answered in this paper concerns the entry labeled '?' in this table: What kind of graph parameter corresponds to the minimum occupation time (mot) for monotone inert fugitive search? In section 2 we introduce a generalization of bandwidth to tree-like layouts, called treespan, based on what we call ordered tree decompositions. In section 3 we give the formal definition of the parameter $\operatorname{mot}(G)$, and then in section 4 we show that it is equivalent to a parameter arising from elimination trees, as used in the sparse matrix computation community. In section 5 we obtain the equivalence also between this elimination tree parameter and treespan, thereby showing that the entry labeled '?' above indeed corresponds to a natural generalization of bandwidth to partially ordered (tree) layouts. In section 6 we obtain some algorithmic and complexity results on the treespan parameter. In section 7 we conclude with some discussion and three open problems.

\section{Motivation through tree decompositions}

We assume simple, undirected, connected graphs $G=(V, E)$, where $|V|=n$. We let $N(v)$ denote the neighbors of vertex $v$, and $d(v)=|N(v)|$ is the degree of $v$. For a set of vertices $U \subseteq V, N(U)=\{v \notin U \mid u v \in E$ and $u \in U\}$. $H \subseteq G$ means that $H$ is a subgraph of $G$. For a rooted tree $T$ and a vertex $v$ in $T$, we let $T[v]$ denote the subtree of $T$ with root in $v$.

A chord of a cycle $C$ in a graph is an edge that connects two non-consecutive vertices of $C$. A graph $G$ is chordal if every cycle of length $\geq 4$ in $G$ has a chord. Given any graph $G=(V, E)$, a triangulation $G^{+}=\left(V, E^{+}\right)$of $G$ is a chordal graph such that $E \subseteq E^{+}$.

A tree decomposition of a graph $G=(V, E)$ is a pair $(X, T)$, where $T=$ $(I, M)$ is a tree and $X=\left\{X_{i} \mid i \in I\right\}$ is a collection of subsets of $V$ called bags, such that: 
1. $\bigcup_{i \in I} X_{i}=V$

2. $u v \in E \Rightarrow \exists i \in I$ with $u, v \in X_{i}$

3. For all vertices $v \in V$, the set $\left\{i \in I \mid v \in X_{i}\right\}$ induces a connected subtree of $T$.

The width of a tree decomposition $(X, T)$ is $t w(X, T)=\max _{i \in I}\left|X_{i}\right|-1$. The treewidth of a graph $G$ is the minimum width over all tree decompositions of $G$. A path decomposition is a tree decomposition $(X, T)$ such that $T$ is a path. The pathwidth of a graph $G$ is the minimum width over all path decompositions of $G$. We refer to Bodlaender's survey [5] for further information on treewidth.

For a chordal graph $G$, the treewidth is one less than the size of the largest clique in $G$. For a non-chordal graph $G$, the treewidth is the minimum treewidth over all triangulations of $G$. This is due to the fact that a tree decomposition $(X, T)$ of $G$ actually corresponds to a triangulation of the given graph $G$ : simply add edges to $G$ such that each bag of $X$ becomes a clique. The resulting graph, which we will call $\operatorname{tri}(X, T)$ is a chordal graph of which $G$ is a subgraph. In addition, any triangulation $G^{+}$of $G$ is equal to $\operatorname{tri}(X, T)$ for some tree decomposition $(X, T)$ of $G$.

Another reason why tree decompositions and chordal graphs are closely related is that chordal graphs are exactly the intersection graphs of subtrees of a tree [16]. Analogously, interval graphs are related to path decompositions, and they are the intersection graphs of subpaths of a path. A graph is interval if there is a mapping $f$ of its vertices into sets of consecutive integers such that for each pair of vertices $v, w$ the following is true: $v w$ is an edge $\Leftrightarrow$ $f(v) \cap f(w) \neq \emptyset$. Interval graphs form a subclass of chordal graphs. Similar to treewidth, the pathwidth of a graph $G$ is one less than the smallest clique number over all triangulations of $G$ into interval graphs.

The bandwidth of $G, b w(G)$, is defined as the minimum, over all linear orders of the vertices of $G$, maximum difference between labels of two adjacent vertices. Similar to pathwidth and treewidth, bandwidth can be defined in terms of triangulations as follows. A graph isomorphic to $K_{1,3}$ is referred to as a claw, and a graph that does not contain an induced claw is said to be claw-free. An interval graph $G$ is a proper interval graph if it is claw-free [26]. As it was observed by Parra \& Scheffler [24], the bandwidth of a graph $G$ is one less than the smallest clique number over all triangulations of $G$ into proper interval graphs. One can define bandwidth in terms of ordered path decompositions. In an ordered path decomposition, the bags are numbered $1,2, \ldots, n$ from left to right. The first bag $X_{1}$ contains only one vertex of $G$, and for $1 \leq i \leq n-1$ we have $\left|X_{i+1} \backslash X_{i}\right|=1$, meaning that exactly one new graph vertex is introduced in each new bag. The number of bags a vertex $v$ belongs to is denoted by $l(v)$. It is easy to show that $b w(G)$ is the minimum, over all ordered path decompositions, $\max \{l(v)-1 \mid v \in V\}$. 
The natural question here is, what kind of parameter corresponds to bandwidth when, instead of path decompositions, we switch to tree decompositions? This brings us to the definition of ordered tree decomposition and treespan.

Definition 2.1. An ordered tree decomposition $(X, T, r)$ of a graph $G=(V, E)$ is a tree decomposition $(X, T)$ of $G$ where $T=(I, M)$ is a rooted tree with root $r \in I$, such that:

$\left|X_{r}\right|=1$, and if $i$ is the parent of $j$ in $T$, then $\left|X_{j} \backslash X_{i}\right|=1$.

Definition 2.2. Given a graph $G=(V, E)$ and an ordered tree decomposition $(X, T, r)$ of $G$, we define:

$l(v)=\left|\left\{i \in I \mid v \in X_{i}\right\}\right|$ (number of bags that contain $v$ ), for each $v \in V$. $t s(X, T, r)=\max \{l(v) \mid v \in V\}-1$.

The treespan of a graph $G$ is $\operatorname{ts}(G)=\min \{t s(X, T, r) \mid(X, T, r)$ is an ordered tree decomposition of $G$ \}.

Since every ordered path decomposition is an ordered tree decomposition, it is clear that for every graph $G, t s(G) \leq b w(G)$.

\section{Search minimizing occupation time with inert fugi- tive}

In this section we give a formal definition of minimum occupation time for inert fugitive searching. A search program $\Pi$ on a graph $G=(V, E)$ is the sequence of pairs

$$
\left(A_{0}, Z_{0}\right),\left(A_{1}, Z_{1}\right), \ldots,\left(A_{m}, Z_{m}\right)
$$

such that

I. For $i \in\{0, \ldots, m\}, A_{i} \subseteq V$ and $Z_{i} \subseteq V$. We say that vertices $A_{i}$ are cleared, vertices $V-A_{i}$ are contaminated and vertices $Z_{i}$ are occupied by searchers at the $i$ th step.

II. (Initial state.) $A_{0}=\emptyset$ and $Z_{0}=\emptyset$. All vertices are contaminated.

III. (Final state.) $A_{m}=V$ and $Z_{m}=\emptyset$. All vertices are cleared.

IV. (Placing-removing searchers and clearing vertices.) For $i \in\{1, \ldots, m\}$ there exists $v \in V$ and $Y_{i} \subseteq A_{i-1}$ such that $A_{i}-A_{i-1}=v$ and $Z_{i}=Y_{i} \cup$ $\{v\}$. Thus at every step one of the searchers is placed on a contaminated vertex $v$ while the others are placed on cleared vertices $Y_{i}$. The searchers are removed from vertices $Z_{i-1}-Y_{i}$. Note that $Y_{i}$ is not necessarily a subset of $Z_{i-1}$. This means that the searchers placed on $Y_{i}$ 'guard' vertices from possible recontamination and the searcher placed on $v$ that 'attacks' the contaminated area. 
V. (Possible recontamination.) For $i \in\{1, \ldots, m\} A_{i}-\{v\}$ is the set of vertices $u \in A_{i-1}$ such that every uv-path has an internal vertex in $Z_{i}$. This means that the fugitive awakening in $v$ can run to a cleared vertex $u$ if there is a $u v$-path unguarded by searchers.

Dendris, Thilikos \& Kirousis [7] initiated the study of inert search problem, where the problem is to find a search program $\Pi$ with the smallest $\max _{i \in\{0, \ldots, m\}}$ $\left|Z_{i}\right|$ (this maximum can be treated as the maximum number of searchers used in one step). It turns out that this number is equal to the treewidth of a graph plus one. The definition of inert search given in [7] is different from ours but it is easy to check the equivalence between both definitions. However the definition we use makes it more easy to define the search cost and other modifications of searching. Thus for example, we find an alternative measure of search to be interesting as well. For a search program $\Pi=\left(A_{0}, Z_{0}\right),\left(A_{1}, Z_{1}\right), \ldots,\left(A_{m}, Z_{m}\right)$ on a graph $G=(V, E)$ and vertex $v \in V$ we define

$$
\delta_{i}(v)=\left\{\begin{array}{l}
1, v \in Z_{i} \\
0, v \notin Z_{i}
\end{array}\right.
$$

Then the number $\sum_{i=0}^{m} \delta_{i}(v)$ is the number of steps at which vertex $v$ was occupied by searchers. For a program $\Pi$ we define the maximum vertex occupation time to be $\operatorname{ot}(\Pi, G)=\max _{v \in V} \sum_{i=0}^{m} \delta_{i}(v)$. The vertex occupation time of a graph $G$, denoted by $o t(G)$, is the minimum maximum vertex occupation time over all search programs on $G$.

A search program $\left(A_{0}, Z_{0}\right),\left(A_{1}, Z_{1}\right), \ldots,\left(A_{m}, Z_{m}\right)$ is monotone if $A_{i-1} \subseteq$ $A_{i}$ for each $i \in\{1, \ldots, m\}$. Note that recontamination does not occur when a searcher is placed on a contaminated vertex thus awaking the fugitive.

Finally, for a graph $G$ we define $\operatorname{mot}(G)$ to be the minimum maximum vertex occupation time over all monotone search programs on $G$. We do not know whether $\operatorname{mot}(G)=\operatorname{ot}(G)$ for every graph $G$, and leave it as an interesting open question.

\section{Searching and elimination trees}

In this section we discuss a relation between $\operatorname{mot}(G)$ and elimination trees of $G$. This relation is not only interesting in its own but also serves as a tool in further proofs.

For a graph $G=(V, E)$, an elimination order $\alpha:\{1,2, \ldots, n\} \rightarrow V$ is a linear order of the vertices of $G$. For each given order $\alpha$, a unique triangulation of $G$ can be computed from the following procedure: starting with vertex $\alpha(1)$, at each step $i$, turn the set of neighbors of vertex $\alpha(i)$ with numbers $>i$ in the transitory graph into a clique by adding edges. The resulting graph, which is denoted by $G_{\alpha}^{+}$, is chordal [14], and the given elimination ordering defines the resulting triangulation. The following lemma follows from the definition of $G_{\alpha}^{+}$. (See also Lemma 1 in [7] for similar results.) 
Lemma 4.1. Edge uv is an edge of $G_{\alpha}^{+}$if and only if $u v$ is an edge of $G$ or there is a path $u, x_{1}, x_{2}, \ldots, x_{k}, v$ in $G$ with $k \geq 1$ such that all $x_{i}$ are ordered before $u$ and $v$ by $\alpha$ (in other words, $\max \left\{\alpha^{-1}\left(x_{i}\right) \mid 1 \leq i \leq k\right\}<$ $\left.\min \left\{\alpha^{-1}(u), \alpha^{-1}(v)\right\}\right)$.

Definition 4.2. Let $\alpha$ be an elimination order on $G$. For a vertex $u$ of $G$ we define $\operatorname{madj}^{+}(u)$ to be $\left\{v \mid \alpha^{-1}(v)>\alpha^{-1}(u)\right.$ and $u v$ is an edge of $\left.G_{\alpha}^{+}\right\}$.

Given a graph $G=(V, E)$, and an elimination order $\alpha$ on $G$, the corresponding elimination tree $E T$ is a rooted tree on the same vertex set, whose edges are defined by the following parent function: parent $(\alpha(i))=\alpha(j)$ where $j=\min \left\{k \mid \alpha(k) \in \operatorname{madj}^{+}(\alpha(i))\right\}$, for $i \in\{1,2, \ldots, n\}$. Hence the elimination tree is a tree on the vertices of $G$, the parent of a vertex $u$ is the lowest numbered vertex among $u$ 's higher numbered neighbors in $G_{\alpha}^{+}$, and vertex $\alpha(n)$ is always the root. For a vertex $v \in V$ we denote by $E T[v]$ the subtree of $E T$ rooted in $v$ and containing all descendants in $E T$ of $v$. It has been shown that if $v \in \operatorname{madj}^{+}(u)$ then $u \in E T[v][25]$. As a consequence, for two vertices $u$ and $v$ such that $E T[u]$ and $E T[v]$ are disjunct subtrees of $E T$, no vertex belonging to $E T[u]$ is adjacent to any vertex belonging to $E T[v]$ in $G$ or $G_{\alpha}^{+}$. We refer to the survey by Liu [21] for further details on elimination trees.

Definition 4.3. Given an elimination tree $E T$ of $G$, the pruned subtree with root in $v, E T_{p}[v]$, is the subtree obtained from $E T[v]$ by deleting the following set of vertices: $\{u \in E T[v] \mid$ neither $u$ nor any descendant of $u$ is a neighbor of $v$ in $G\}$.

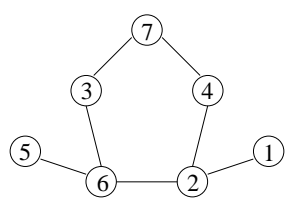

a)

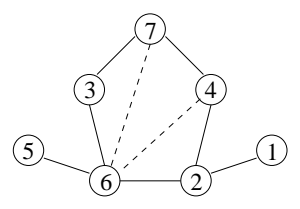

b)

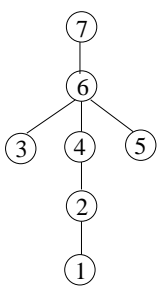

c)

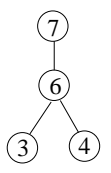

d)

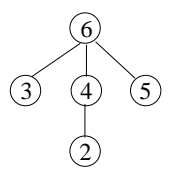

e)

Figure 1: A graph $G$ with a given order $\alpha$ is shown in a), and b) shows $G_{\alpha}^{+}$. The corresponding elimination tree $E T$ and the pruned subtrees $E T_{p}[7]$ and $E T_{p}[6]$ are given in c), d), and e), respectively.

An example to illustrate this definition is given in Figure 1. It follows from Definition 4.3 that the leaves of $E T_{p}[v]$ are neighbors of $v$ in $G$. In addition, there might clearly appear vertices in $E T_{p}[v]$ that are not neighbors of $v$ in $G$. However, every vertex that appears in $E T_{p}[v]$ is a neighbor of $v$ in $G^{+}$, by the following result.

Theorem 4.4. [20] Let $\alpha$ be an elimination order of $G=(V, E)$ and let $E T$ be a corresponding elimination tree. Then for any $u, v \in V$ with $\alpha^{-1}(u)<$ 
$\alpha^{-1}(v), v \in \operatorname{madj}^{+}(u)$ if and only if $v$ has a neighbor $w$ in $G$ such that $w \in E T[u]$.

As a direct consequence, $E T_{p}[v]$ contains, in addition to $v$, exactly the set of lower numbered neighbors of $v$ in $G_{\alpha}^{+}$, as stated in the following.

Corollary 4.5. Let $\alpha$ be an elimination order of graph $G=(V, E)$ and let ET be a corresponding elimination tree. Then for any distinct $u, v \in V, u \in E T_{p}[v]$ if and only if $v \in \operatorname{madj}^{+}(u)$. Hence $E T_{p}[v]=\left\{u \mid v \in \operatorname{madj}^{+}(u)\right\} \cup\{v\}$

We define a parameter called elimination span, es, as follows:

Definition 4.6. Given an elimination tree $E T$ of a graph $G=(V, E)$, for each vertex $v \in V$ we define $s(v)=\left|E T_{p}[v]\right|$ and $\operatorname{es}(E T)=\max \{s(v) \mid v \in$ $V\}-1$. The elimination span of a graph $G$ is $\operatorname{es}(G)=\min \{\operatorname{es}(E T) \mid E T$ is an elimination tree of $G\}$.

Theorem 4.7. For any graph $G=(V, E)$, es $(G)=\operatorname{mot}(G)-1$.

Proof. Let us prove $e s(G) \leq \operatorname{mot}(G)-1$ first. Let

$$
\Pi=\left(A_{0}, Z_{0}\right),\left(A_{1}, Z_{1}\right), \ldots,\left(A_{m}, Z_{n}\right)
$$

be a monotone search program. At every step of the program exactly one new vertex $A_{i}-A_{i-1}$ is cleared. Thus we can define the elimination ordering $\alpha$ by putting for $1 \leq i \leq n$

$$
\alpha\left(A_{i}-A_{i-1}\right)=n-i+1 .
$$

At the $i$ th step, when a searcher is placed at a vertex $u=A_{i}-A_{i-1}$ every vertex $v \in A_{i}$ such that there is a $u v$-path with no inner vertices in $A_{i}$ should be occupied by a searcher (otherwise $v$ would be recontaminated). Therefore, if $v \in \operatorname{madj}^{+}(u)$ then $v$ is a occupied by a searcher during the step when a searcher is placed at $u$ and the number of steps when vertex $v$ is occupied by searchers, is at least $\left|\left\{u \mid v \in \operatorname{madj}^{+}(u)\right\} \cup\{v\}\right|$. By Corollary 4.5, $\mid\{u \mid v \in$ $\left.\operatorname{madj}^{+}(u)\right\} \cup\{v\} \mid=s(v)$ and we arrive at

$$
e s(E T) \leq \operatorname{mot}(\Pi, G)-1,
$$

where $E T$ is the elimination tree corresponding to the ordering $\alpha$.

We now show that $e s(G) \geq \operatorname{mot}(G)-1$. Let $E T$ be an elimination tree and let $\alpha$ be a corresponding elimination ordering. We consider a search program $\Pi$ where at the $i$ th step of the program, $1 \leq i \leq n$, the searchers occupy the set of vertices $\operatorname{madj}^{+}(v) \cup\{v\}$, where $v$ is a vertex with $\alpha(v)=n-i+1$. Let us first prove that $\Pi$ is recontamination free. Suppose, on the contrary, that a vertex $u$ is recontaminated at the $i$ th step after placing a searcher on a vertex $v$. Then there is a $u v$-path $P$ such that no vertex of $P$ except $v$ contains a searcher at the $i$ th step. On the other hand, vertex $u$ is after $v$ in ordering $\alpha$. Thus $P$ should contain a vertex $w \in \operatorname{madj}^{+}(v)$, occupied 
by a searcher. This is a contradiction. Since every vertex was occupied at least once and no recontamination occurs, we conclude that at the end of $\Pi$ all vertices are cleared. Every vertex $v$ was occupied by searchers during $\left|\left\{u \mid v \in \operatorname{madj}^{+}(u)\right\} \cup\{v\}\right|$ steps and using Corollary 4.5 we conclude that $\operatorname{es}(E T) \geq \operatorname{mot}(\Pi, G)-1$.

\section{$5 \quad$ Ordered tree decompositions and elimination trees}

In this section we discuss a relation between the treespan $t s(G)$ and elimination trees of $G$, establishing that $\operatorname{ts}(G)=\operatorname{mot}(G)-1$. We first give a simplified view of ordered tree decompositions and then proceed to prove some of their properties.

There are exactly $n$ bags in $X$ of an ordered tree decomposition $(X, T, r)$ of $G$. Thus, the index set $I$ for $X_{i}, i \in I$ can be chosen so that $I=V$, with $r \in V$. Then $T$ is a tree on the vertices of $G$. To identify the bags and to define the correspondence between $I$ and $V$ uniquely, name the bags so that $X_{r}$ is the bag corresponding to the root $r$ of $T$. Regarding the bags in a top down fashion according to $T$, name the bag in which vertex $v$ appears for the first time $X_{v}$ and the corresponding tree node $v$. Thus if $y$ is the parent of $v$ in $T$ then $X_{v} \backslash X_{y}=\{v\}$. This explains how to rename the bags and the vertices of $T$ with elements from $V$ given a tree decomposition based on $I$. However, if we replace $i$ with $v$ and $I$ with $V$ in Conditions $1-3$ of the definition of a tree decomposition, and change condition in the definition of ordered tree decompositions to " $X_{r}=\{r\}$, and if $y$ is the parent of $v$ in $T$ then $X_{v} \backslash X_{y}=\{v\}$ ", then this will automatically give a tree $T$ on the vertices of $G$ as we have explained above. For the remainder of this paper, when we mention an ordered tree decomposition $(X, T, r)$, we will assume that $T$ is a tree on the vertices of $G$ as explained here. The following lemma will make the role of $T$ even clearer.

Lemma 5.1. Let $T$ be a rooted tree on the vertex set of a graph $G=(V, E)$. There exists an ordered tree decomposition $(X, T, r)$ of $G$ if and only if for every edge $u v \in E, u$ and $v$ have an ancestor-descendant relationship in $T$.

Proof. Assume that $T$ corresponds to an ordered tree decomposition of $G$, but there is an edge $u v$ in $G$ such that $T[u]$ and $T[v]$ are disjunct subtrees of T. $X_{u}$ is the first bag in which $u$ appears and $X_{v}$ is the first bag in which $v$ appears, thus $u$ and $v$ do not appear in any bag $X_{w}$ where $w$ is on the path from $u$ to the root or from $v$ to the root in $T$. Thus if $u$ and $v$ appear together in any other bag $X_{y}$ where $y$ belongs to $T[u]$ or $T[v]$ or any other disjunct subtree in $T$, this would violate Condition 3 of a tree decomposition. Therefore, $u$ and $v$ cannot appear together in any bag, and there cannot exist a valid decomposition $(X, T, r)$ of $G$.

For the reverse direction, assume that for every edge $u v$ in $G, u$ and $v$ have an ancestor-descendant relationship in $T$. Assume without loss of generality 
that $v$ is an ancestor of $u$. Then the bags can be defined so that 1) $X_{v}$ contains $v, 2)$ no bag $X_{y}$ contains $v$ where $y$ is an ancestor of $v, 3$ ) for every vertex $w$ on the path from $v$ to $u$ in $T, X_{w}$ contains $v$ (and $w$ of course), and 4) $X_{u}$ contains both $u$ and $v$. We can see that all the conditions of an ordered tree decomposition are satisfied.

Lemma 5.2. For every graph $G$, there exists an ordered tree decomposition $(X, T, r)$ of $G$ of minimum treespan such that if $u$ is a child of $v$ in $T$ then $v \in X_{u}$.

Proof. Assume that $u$ is a child of $v$ in $T$ and $v \notin X_{u}$. By the properties of tree decompositions, $v$ does not belong to any bag $X_{y}$ where $y$ is a descendant of $u$, and thus $X_{v}$ is the only bag that contains $v$. By the definition of ordered tree decompositions, neither $u$ nor any descendant of $u$ belongs to $X_{v}$. As a consequence, neither $u$ nor any descendant of $u$ in $T$ is a neighbor of $v$ in $G$. Since $G$ is connected, $u$ or a descendant of $u$ must have a neighbor $w$ in $G$ such that $w$ is an ancestor of $v$ in $T$. Let $w$ be such a node of $T$ that is closest to $v$. Thus no descendant of $w$ is adjacent in $G$ to $u$ or any descendant of $u$. Observe that $w$ must belong to $X_{u}$. We now change the tree decomposition as follows: remove edge $v u$ from $T$, add edge $w u$ to $T$, and remove $w$ from any $\operatorname{bag} X_{z}$ that it can be removed from, where $z=v$ or $z$ is on the path between $v$ and $w$ in $T$. This operation results in a new ordered tree decomposition, it does not increase $l(w)$, and for every other vertex $x, l(x)$ is unchanged. This can be repeated to achieve an ordered tree decomposition as claimed.

Lemma 5.3. Let $(X, T, r)$ be an ordered tree decomposition of a given graph. For every edge uv in tri $(X, T), u$ and $v$ have an ancestor-descendant relationship in $T$.

Proof. As we have seen in the proof of Lemma 5.1, if $u$ and $v$ belong to disjunct subtrees of $T$, then they cannot appear together in the same bag. Since only the bags are made into cliques, $u$ and $v$ cannot belong to the same clique in $\operatorname{tri}(X, T)$, which means that the edge $u v$ does not exist in $\operatorname{tri}(X, T)$.

Lemma 5.4. Let $(X, T, r)$ be an ordered tree decomposition of a given graph. Let $u v$ be an edge of tri $(X, T)$ such that $v$ is an ancestor of $u$ in $T$. Then $v$ belongs to bag $X_{w}$ for every $w$ on the path from $v$ to $u$ including $X_{v}$ and $X_{u}$.

Proof. Vertex $v$ appears for the first time in $X_{v}$ on the path from the root, and $u$ appears for the first time in $X_{u}$. For every vertex $w$ on the path from $v$ to $u$, exactly vertex $w$ is introduced in $X_{w}$. Thus $X_{u}$ is the first bag in which $u$ and $v$ both can belong to. In order for this to be possible, $v$ must belong to bag $X_{w}$ for every vertex $w$ on the path from $v$ to $u$ in $T$.

Lemma 5.5. Let $(X, T, r)$ be an ordered tree decomposition of $G$, and let $\alpha:\{1, \ldots, n\} \rightarrow V$ be a post order of $T$. Then $G_{\alpha}^{+} \subseteq \operatorname{tri}(X, T)$. 
Proof. Let $u v$ be an edge of $G_{\alpha}^{+}$, and assume without loss of generality that $u$ has a lower number than $v$ according to $\alpha$. If $u v$ is an edge of of $G$, then we are done. Otherwise, due to Lemma 4.1, there must exist a path $u, x_{1}, x_{2}, \ldots, x_{k}, v$ in $G$ with $k \geq 1$ such that all $x_{i}$ are ordered before $u$. Since $\alpha$ is a post order of $T$, none of the vertices $x_{i}, i=1, \ldots, k$, can lie on the path from $u$ to the root in $T$. Consequently and due to Lemma 5.1 , since $u x_{1}$ is an edge of $G, x_{1}$ belongs to $T[u]$. With the same argument, since $x_{1}, x_{2}, \ldots, x_{k}$ is a path in $G$, all the vertices $x_{1}, x_{2}, \ldots, x_{k}$ must belong to $T[u]$. Now, since $v x_{k}$ is an edge in $G, v$ must be an ancestor of $x_{k}$ and thus of $u$ in $T$, where $u$ lies on the path from $v$ to $x_{k}$. By Lemma 5.4, vertex $v$ must be present in all bags $X_{w}$ where $w$ lies on the path from $v$ to $x_{k}$, and consequently also in bag $X_{u}$. Therefore, $u$ and $v$ are both present in bag $X_{u}$ and are neighbors in $\operatorname{tri}(X, T)$.

Lemma 5.6. Let $(X, T, r)$ be an ordered tree decomposition of $G$, and let $\alpha$ be a post order of $T$. Let ET be the elimination tree of $G$ corresponding to elimination order $\alpha$. Then for any vertex $u$, if $v$ is the parent of $u$ in ET, then $v$ lies on the path from $u$ to the root in $T$.

Proof. Since $v$ is the parent of $u$ in ET, $u v$ is an edge of $G_{\alpha}^{+}$. By Lemma $5.5, u v$ is also an edge of $\operatorname{tri}(X, T)$. By Lemma 5.3, $u$ and $v$ must have an ancestor-descendant relationship in $T$. Since $\alpha$ is a post order of $T$, and $\alpha^{-1}(u)<\alpha^{-1}(v), v$ must be an ancestor of $u$ in $T$.

Theorem 5.7. For any graph $G, t s(G)=e s(G)$.

Proof. First we prove that $\operatorname{ts}(G) \leq e s(G)$. Let $E T$ be an elimination tree of $G=(V, E)$ such that $e s(G)=e s(E T)$, and let $r$ be the root vertex of $E T$. We define an ordered tree decomposition $\left(X=\left\{X_{v} \mid v \in V\right\}, T=E T, r\right)$ of $G$ in the following way. For each vertex $v$ in $E T$, put $v$ in exactly the bags $X_{u}$ such that $u \in E T_{p}[v]$. Regarding $E T$ top down, each vertex $u$ will appear for the first time in bag $X_{u}$, and clearly $\left|X_{u} \backslash X_{v}\right|=1$ whenever $v$ is the parent of $u$. It remains to show that $(X, E T)$ is a tree decomposition of $G$. Conditions 1 and 3 of a tree decomposition are trivially satisfied since for every vertex $v$ bag $X_{v}$ contains $v$ and $E T_{p}[v]$ is connected. For Condition 2, if $u v$ is an edge of $G$, then the lower numbered of $v$ and $u$ is a descendant of the other in ET. Let us say $u$ is a descendant of $v$, then $u \in E T_{p}[v]$, and $v$ and $u$ will both appear in bag $X_{u}$. Thus $(X, E T)$ is an ordered tree decomposition of $G$, and clearly, $t s(X, E T)=e s(G)$. Consequently, $t s(G) \leq e s(G)$.

Now we show that $e s(G) \leq t s(G)$. Let $(X, T, r)$ be an ordered tree decomposition of $G$ with $t s(X, T, r)=t s(G)$. Let $\alpha$ be a post order on $T$, and let $E T$ be the elimination tree of $G$ corresponding to elimination order $\alpha$. For any two adjacent vertices $u$ and $v$ in $G, u$ and $v$ must have an ancestor-descendant relationship both in $T$ and in ET. Moreover, due to Lemma 5.6, all vertices that are on the path between $u$ and $v$ in ET must also be present on the path between $u$ and $v$ in $T$. Assume, without loss of generality, that $u$ is numbered 
lower than $v$. By Lemma 5.4, $v$ must belong to all the bags corresponding to the vertices on the path from $v$ to $u$ in $T$. Thus for each vertex $v, s(v)$ in ET is at most $l(v)$ in $(X, T, r)$. Consequently, es $(G) \leq t s(G)$, and the proof is complete.

Theorems 4.7 and 5.7 imply the main combinatorial result of this paper.

Corollary 5.8. For any graph $G, t s(G)=e s(G)=\operatorname{mot}(G)-1$.

\section{Treespan of some special graph classes}

The diameter of a graph $G$, $\operatorname{diam}(G)$, is the maximum length of a shortest path between any two vertices of $G$. The density of a graph $G$ is defined as $\operatorname{dens}(G)=(n-1) / \operatorname{diam}(G)[6]$. The following result is well known

Lemma 6.1. [6] For any graph $G, b w(G) \geq \max \{\operatorname{dens}(H) \mid H \subseteq G\}$.

A caterpillar is a tree consisting of a main path of vertices of degree at least two with some leaves attached to the vertices of the main path.

Theorem 6.2. For any graph $G, t s(G) \geq \max \{\operatorname{dens}(H) \mid H \subseteq G$ and $H$ is a caterpillar\}.

Proof. Let the caterpillar $H$ be a subgraph of $G$ consisting of the following main path: $c_{1}, c_{2}, \ldots, c_{\text {diam }(H)-1}$. We view the bags of an ordered tree decomposition as labeled by vertices of $G$ in the natural manner (as described before Lemma 5.1). Let $(X, T, r)$ be an ordered tree decomposition of $G$ with $\left(X^{\prime}, T^{\prime}, r^{\prime}\right)$ being the topologically induced ordered tree decomposition on $H$, i.e. containing only bags labeled by a vertex from $H$, where we contract edges of $T$ going to vertices labeled by vertices not in $H$ to get $T^{\prime}$. Let $X_{c_{i}}$ be the 'highest' bag in $\left(X^{\prime}, T^{\prime}, r^{\prime}\right)$ labeled by a vertex from the main path, so that only the subtree of $\left(X^{\prime}, T^{\prime}, r^{\prime}\right)$ rooted at $X_{c_{i}}$ contains any vertices from the main path. Let there be $h+1$ bags on the path from $X_{c_{i}}$ to the root $X_{r^{\prime}}$ of $\left(X^{\prime}, T^{\prime}, r^{\prime}\right)$. Since vertex $r^{\prime}$ of $H$ (a leaf unless $r^{\prime}=c_{i}$ ) is adjacent to a vertex on the main path it appears in at least $h+1$ bags, giving $t s(G) \geq h$. Moreover, by applying Lemma 5.2 we get that $T^{\prime}$ between its root $X_{r^{\prime}}$ and $X_{c_{i}}$ consists simply of a path without further children, so that the subtree rooted at $X_{c_{i}}$ has $|V(H)|-h$ bags. Each of these bags contain a vertex from the main path since every leaf of $H$ is adjacent in $H$ only to a vertex on the main path, and by the pigeonhole principle we thus have that some main path vertex is in at least $\lceil(|V(H)|-h) /(\operatorname{diam}(H)-1)\rceil$ bags. If $(|V(H)|-h) /(\operatorname{diam}(H)-1)$ is not an integer, then immediately we have the bound $t s(G) \geq\lfloor(|V(H)|-h) /(\operatorname{diam}(H)-1)\rfloor$. If $(\operatorname{diam}(H)-1)$ on the other hand does divide $(|V(H)|-h)$ then we apply the fact that at least $\operatorname{diam}(H)-2$ bags must contain at least two vertices from the main path, to account for edges between them, and for $\operatorname{diam}(H) \geq 3$ (which holds except 
for the trivial case when $H$ is a star) this increases the span of at least one main path vertex and we again get $t s(G) \geq\lfloor(|V(H)|-h) /(\operatorname{diam}(H)-1)\rfloor$.

Thus $t s(G) \geq \max \{h,\lfloor(|V(H)|-h) /(\operatorname{diam}(H)-1)\rfloor\}$. If $h \leq \operatorname{dens}(H)$ we have that $\lfloor(|V(H)|-h) /(\operatorname{diam}(H)-1)\rfloor \geq(|V(H)|-1) / \operatorname{diam}(H)$ and therefore $\lfloor(|V(H)|-h) /(\operatorname{diam}(H)-1)\rfloor \geq \operatorname{dens}(H)$. We conclude that $t s(G) \geq \operatorname{dens}(H)$ and the lemma follows.

With this theorem, in connection with the following result from [2], we can conclude that $b w(G)=t s(G)$ for a caterpillar graph $G$.

Lemma 6.3. [2] For a caterpillar graph $G$, bw $(G) \leq \max \{\operatorname{dens}(H) \mid H \subseteq G\}$.

Lemma 6.4. For a caterpillar graph $G$, bw $(G)=t s(G)=\max \{\operatorname{dens}(H) \mid$ $H \subseteq G\}$.

Proof. Let $G$ be a caterpillar graph. Then, $b w(G) \geq t s(G) \geq \max \{\operatorname{dens}(H) \mid$ $H \subseteq G\} \geq b w(G)$. The first inequality was mentioned in Section 2, the second inequality is due to Theorem 6.2, and the last inequality is due to Lemma 6.3 since $G$ is a caterpillar. Thus all of the mentioned parameters on $G$ are equal.

A set of three vertices $x, y, z$ of a graph $G$ is called an asteroidal triple (AT) if for any two of these vertices there exists a path joining them that avoids the (closed) neighborhood of the third. A graph $G$ is called an asteroidal triple-free (AT-free) graph if $G$ does not contain an asteroidal triple. This notion was introduced by Lekkerkerker an Boland [19] for the following characterization of interval graphs: $G$ is an interval graph if and only if it is chordal and ATfree. A graph isomorphic to $K_{1,3}$ is referred to as a claw, and a graph that does not contain an induced claw is said to be claw-free.

A graph $G$ is said to be cobipartite if it is the complement of a bipartite graph. Notice that cobipartite graphs form a subclass of AT-free claw-free graphs. Another subclass of AT-free claw-free graphs are the proper interval graphs, which were mentioned earlier. Thus $G$ is a proper interval graph if and only if it is chordal and AT-free claw-free. A minimal triangulation of $G$ is a triangulation $H$ such that no proper subgraph of $H$ is a triangulation of $G$. The following result is due to Parra and Scheffler.

Theorem 6.5. [24] Let $G$ be an AT-free claw-free graph. Then every minimal triangulation of $G$ is a proper interval graph, and hence, bw $(G)=p w(G)=$ $t w(G)$.

Theorem 6.6. For an AT-free claw-free graph $G, t s(G)=b w(G)=p w(G)=$ $t w(G)$. 
Proof. Let $G$ be AT-free claw-free and let $H$ be its minimal triangulation such that $t s(G)=t s(H)$. Such a graph $H$ must exist, since for an optimal ordered tree decomposition $(X, T, r)$, the graph $\operatorname{tri}(X, T)$ is chordal and $\operatorname{ts}(\operatorname{tri}(X, T))=\operatorname{ts}(G)$. Thus any minimal graph from the set of chordal graphs 'sandwiched' between $\operatorname{tri}(X, T)$ and $G$ can be chosen as $H$. By Theorem $6.5, H$ is a proper interval graph. It is well known [24] that the maximum clique size $\omega(H)$ of a proper interval graph is equal to $b w(H)+1$. Thus $\omega(H)-1=b w(H) \geq b w(G)$. Since $t s(H) \geq \omega(H)-1$, we have that $t s(G)=t s(H) \geq \omega(H)-1 \geq b w(G) \geq t s(G)$.

By the celebrated result of Arnborg, Corneil \& Proskurowski [1], treewidth (and hence path-width and bandwidth) is NP-hard even for cobipartite graphs. Thus Theorem 6.6 yields the following corollary.

Corollary 6.7. Computing treespan is NP-hard for cobipartite graphs.

\section{Open problems and concluding remarks}

We have introduced a new graph parameter called treespan, that can be seen as a generalization of bandwidth to tree layouts, with connections also to elimination trees as used in sparse matrix computations, but with the main viewpoint emphasized in this paper being its role in inert graph searching. The most important and challenging open question about this new parameter is if recontamination can help or if we may as well assume monotonicity, in other words if $o t(G)=\operatorname{mot}(G)$ for any graph $G$.

Another interesting version of inert searching is when instead of minimizing vertex occupation time one wants to minimize the 'average' vertex occupation time

$$
\sum_{i=0}^{m}\left|Z_{i}\right|
$$

as indicated by the column labeled 'Cost of Searching' in the Table given in Section 1. As promised in that section we now show that this version of monotone searching is related to the fill-in problem of adding the smallest number of edges to get a chordal supergraph. This result was obtained by Fomin, Stamatiou \& Thilikos [11], we give the proof here for completeness. Our second open problem is similar to the first: can recontamination help for this version of inert searching?

Theorem 7.1. For any graph $G=(V, E)$ the following are equivalent:

a) There is a chordal supergraph of $G$ with at most $k$ edges;

b) There is a monotone search program (for inert fugitive)

$$
\Pi=\left(A_{0}, Z_{0}\right),\left(A_{1}, Z_{1}\right), \ldots,\left(A_{m}, Z_{m}\right)
$$


such that

$$
\sum_{i=0}^{m}\left|Z_{i}\right| \leq k
$$

Proof. b) $\Rightarrow$ a). Let $\Pi=\left(A_{0}, Z_{0}\right),\left(A_{1}, Z_{1}\right), \ldots,\left(A_{m}, Z_{n}\right)$ be a monotone search program. As in the proof of Theorem 4.7, $\Pi$ defines an elimination order $\alpha$ by putting for $1 \leq i \leq n$

$$
\alpha\left(A_{i}-A_{i-1}\right)=n-i+1 .
$$

The corresponding supergraph $G_{\alpha}^{+}=\left(V, E^{+}\right)$of $G$ is chordal and

$$
\left|E^{+}\right|=\sum_{v \in V}\left|\operatorname{madj} j^{+}(v)\right|
$$

As in Theorem 4.7, the number of steps when a vertex $v$ is occupied by searchers, is $\left|\left\{u \mid v \in \operatorname{madj}^{+}(u)\right\}\right|$. Hence $\sum_{i=0}^{m}\left|Z_{i}\right|=\sum_{v \in V} \mid\{u \mid v \in$ $\left.\operatorname{madj}^{+}(u)\right\} \mid$. Since

$$
\sum_{v \in V}\left|\left\{u \mid v \in \operatorname{madj}^{+}(u)\right\}\right|=\sum_{v \in V}\left|\operatorname{madj}^{+}(v)\right|,
$$

we have that

$$
\sum_{i=0}^{m}\left|Z_{i}\right|=\left|E^{+}\right|
$$

a) $\Rightarrow$ b). Let $\alpha$ be an elimination ordering of $G$ and let $G_{\alpha}^{+}=\left(V, E^{+}\right)$be the corresponding chordal supergraph of $G$. As in the proof of Theorem 4.7, we construct a monotone search program

$$
\Pi=\left(A_{0}, Z_{0}\right),\left(A_{1}, Z_{1}\right), \ldots,\left(A_{m}, Z_{n}\right)
$$

such that for $1 \leq i \leq n$ the searchers occupy the set of vertices $Z_{i}=\operatorname{madj}^{+}(v)$, where $v$ is a vertex with $\alpha(v)=n-i+1$. In the proof of Theorem 4.7 we show that every vertex $v$ was occupied by searchers during $\left|\left\{u \mid v \in \operatorname{madj}^{+}(u)\right\}\right|$ steps. Thus

$$
\sum_{i=0}^{m}\left|Z_{i}\right|=\sum_{v \in V}\left|\left\{u \mid v \in \operatorname{madj}^{+}(u)\right\}\right|=\sum_{v \in V}\left|\operatorname{madj}^{+}(v)\right|=\left|E^{+}\right| .
$$

It is easy to check that for trees of maximum degree at most $3, \operatorname{ts}(G)=$ $\lceil\Delta(G) / 2\rceil$, where $\Delta(G)$ denotes the maximum degree of any vertex in $G$. Notice that bandwidth is NP-complete on trees of max degree 3 [15]. Our third open problem is the question whether treespan can be computed in polynomial time for trees of larger max degree. 


\section{References}

[1] S. Arnborg, D. G. Corneil, and A. Proskurowski, Complexity of finding embeddings in a k-tree, SIAM J. Alg. Disc. Meth., 8 (1987), pp. 277-284.

[2] S. F. Assman, G. W. Peck, M. M. SysŁo, and J. Zak, The bandwidth of caterpillars with hairs of length 1 and 2, SIAM J. Alg. Disc. Meth., 2 (1981), pp. 387-392.

[3] D. BIEnstock, Graph searching, path-width, tree-width and related problems (a survey), DIMACS Ser. in Discrete Mathematics and Theoretical Computer Science, 5 (1991), pp. 33-49.

[4] D. Bienstock And P. Seymour, Monotonicity in graph searching, J. Algorithms, 12 (1991), pp. $239-245$.

[5] H. L. Bodlaender, A partial k-arboretum of graphs with bounded treewidth, Theor. Comp. Sc., 209 (1998), pp. 1-45.

[6] P. Z. Chinn, J. Chvátalová, A. K. Dewdney, and N. E. Gibbs, The bandwidth problem for graphs and matrices - a survey, J. Graph Theory, 6 (1982), pp. 223-254.

[7] N. D. Dendris, L. M. Kirousis, And D. M. Thilikos, Fugitive-search games on graphs and related parameters, Theor. Comp. Sc., 172 (1997), pp. 233-254.

[8] J. A. Ellis, I. H. Sudborough, And J. Turner, The vertex separation and search number of a graph, Information and Computation, 113 (1994), pp. 50-79.

[9] F. Fomin, Helicopter search problems, bandwidth and pathwidth, Discrete Appl. Math., 85 (1998), pp. 59-71.

[10] F. V. Fomin and P. A. Golovach, Graph searching and interval completion, SIAM J. Discrete Math., 13 (2000), pp. 454-464.

[11] F. V Fomin, Y. Stamatiou, and D. M. Thilikos, Unpublished results.

[12] F. V Fomin And D. M. Thilikos, On the Monotonicity of Games Generated by Symmetric Submodular Functions, Discr. Appl. Math. 131 (2003), no. 2, pp. 323335 .

[13] M. Franklin, Z. Galil, And M. Yung, Eavesdropping games: A graphtheoretic approach to privacy in distributed systems, J. ACM, 47 (2000), pp. 225 $-243$.

[14] D. Fulkerson and O. Gross, Incidence matrices and interval graphs, Pacific Journal of Math., 15 (1965), pp. 835-855.

[15] M. R. Garey, R. L. Graham, D. S. Johnson, and D. E. Knuth, Complexity results for bandwidth minimization, SIAM J. Appl. Math., 34 (1978), pp. 477-495.

[16] F. GAVRIL, The intersection graphs of subtrees in trees are exactly the chordal graphs, J. Combin. Theory Ser. B, 16 (1974), pp. 47-56.

[17] L. M. Kirousis and C. H. Papadimitriou, Searching and pebbling, Theor. Comp. Sc., 47 (1986), pp. 205-218. 
[18] A. S. LaPaugh, Recontamination does not help to search a graph, J. ACM, 40 (1993), pp. 224-245.

[19] C. G. LekKerkerker and J. C. Boland, Representation of a finite graph by a set of intervals on the real line, Fund. Math, 51 (1962), pp. 45-64.

[20] J. W. H. LIU, A compact row storage scheme for Cholesky factors using elimination trees, ACM Trans. Math. Software, 12 (1986), pp. 127-148.

[21] J. W. H. LIU, The role of elimination trees in sparse factorization, SIAM J. Matrix Anal. Appl., 11 (1990), pp. 134-172.

[22] F. S. Makedon, C. H. Papadimitriou, and I. H. Sudborough, Topological bandwidth, SIAM J. Alg. Disc. Meth., 6 (1985), pp. 418-444.

[23] F. S. Makedon and I. H. Sudborough, On minimizing width in linear layouts, Disc. Appl. Math., 23 (1989), pp. 201-298.

[24] A. Parra And P. Scheffler, Treewidth equals bandwidth for AT-free claw-free graphs, Technical Report 436/1995, Technische Universität Berlin, Fachbereich Mathematik, Berlin, Germany, 1995.

[25] R. SChreIBer, A new implementation of sparse Gaussian elimination, ACM Trans. Math. Software, 8 (1982), pp. 256-276.

[26] F. S. Roberts, Indifference graphs, in Proof Techniques in Graph Theory, F. Harary, ed., Academic Press, 1969, pp. 139 - 146.

[27] N. Robertson and P. D. Seymour, Graph minors - a survey, in Surveys in Combinatorics, I. Anderson, ed., Cambridge Univ. Press, 1985, pp. 153-171.

[28] A. L. Rosenberg And I. H. Sudborough, Bandwidth and pebbling, Computing, 31 (1983), pp. 115-139. 